\title{
Frozen and paraffin sections - Comparative study highlighting the concordance and discordance rates in a tertiary care centre
}

\author{
Dr.K.Chandramouleeswari ${ }^{1}$, Dr.M.Yogambal ${ }^{2}$, Dr. P. Arunalatha ${ }^{3}$, \\ Dr. Jagadeesh Chandra Bose ${ }^{4}$, Dr. A. Rajendran ${ }^{5}$ \\ ${ }^{\text {I}}$ (Department of pathology, Stanley medical college, The Tamilnadu Dr.M.G.R medical University, India) \\ ${ }^{2}$ (Department of pathology, Stanley medical college, The Tamilnadu Dr.M.G.R medical University, India) \\ ${ }_{3}^{3}$ (Department of pathology, Stanley medical college, The Tamilnadu Dr.M.G.R medical University, India) \\ ${ }^{4}$ (Department of surgical oncology, Madras medical college, The Tamilnadu Dr.M.G.R medical University, \\ India) \\ ${ }^{5}$ (Department of surgery, Stanley medical college, The Tamilnadu Dr.M.G.R medical University, India)
}

\begin{abstract}
Aim: Frozen section is a highly accurate and useful procedure wherein knowledge of the clinical presentation, good rapport with the operating surgeon, pathologist's experience and in depth knowledge of the subject, awareness of the limitations of the procedure, ability to provide a rapid and reliable diagnosis under pressure are of great importance. The correlation between intra operative frozen section diagnoses with final histopathological diagnosis is an integral part of quality assurance in surgical pathology. The indication, methodology, discordance of result, causes for the discordance and deferrals were analysed to improve the accuracy of frozen section diagnosis and to minimise the avoidable errors.

Method: This present study was a retrospective analysis of 51 cases of frozen section biopsy, diagnosed and reported in our tertiary care hospital pathology department over a period of one year. Taking the paraffin section diagnosis as the gold standard, the diagnoses on frozen sections were compared with the final diagnosis on paraffin sections to assess the concordance and discordance rates between both.

Results: The overall concordance rate over a period of one year was $92 \%$, discordance rate was $2 \%$ and deferred diagnosis was $6 \%$ in our institution.

Conclusion: In this study conducted in our tertiary care hospital, the results obtained were similar to those cited in various articles published.Diagnosis made by frozen section is a very useful and accurate procedure provided correct guidelines are followed and its limitations understood.

Key words: Artifact, Frozen section, Histopathology
\end{abstract}

\section{Introduction}

The technique of frozen section was first introduced by the pathologist, William H.Welch, in 1891. In 1920s the technique became popular and was used for intra operative consultation. However, the technical quality of most frozen sections during these years was suboptimal. In the 1960s, the preparation of frozen section was made easier by the use of cryostat, a cabinet with -20 to -30 degree Celsius cooling and enclosing a microtome blade. $[1,2,3,4]$

The main purpose of frozen section is to provide rapid diagnosis to guide the surgeon in per operative decision making. Per operative indications of frozen section are identification of tissue, to establish that sufficient diagnostic tissue has been obtained; identification of lymph node metastasis and to obtain a tissue diagnosis as to the nature of the lesion. [5, 6]The other indications of frozen sections are for performing enzyme histochemistry, immunohistochemistry and in immunofluorescence. Though there are many indications for frozen section, per operative diagnosis and patient management continues to be its foremost and important use in a general pathology and oncology setup.

College of American Pathologist (CAP) sponsored review of over 90,000 frozen section at 461 institutions showed a concordance rate of $98.52 \%$ between frozen and subsequent biopsy diagnosis. This study reasons that the main cause for discrepancies were either misinterpretation of original frozen section (31.8\%), absence of diagnostic tissue in the frozen material but present in the un sampled tissue or in the corresponding permanent sections (31.4\%). [7]

Here, an analysis of the causes for discordant results and deferral showed that many of the errors during a frozen section are avoidable and it is a most valuable and reliable methodology which should be used in the routine surgical pathology practice in a tertiary care centre.

\section{Materials and methods}

This study is a retrospective analysis of 51 cases of frozen section along with their corresponding permanent section, reported in our tertiary care hospital over a period of one year. All the data's were retrieved 
from well preserved registers maintained in our pathology department. The tissues were sent to the pathology laboratory, during the surgical procedure. for an intraoperative diagnosis to be made and frozen sections were carried out using a modern cryostat machine .The slides were stained with haematoxylin and eosin $(\mathrm{H} \mathrm{\&} \mathrm{E)}$ stain and reported based on which recommended management was carried out. Subsequently specimens were fixed in $10 \%$ neutral buffered formalin, grossed and adequate permanent representative sections were taken according to the standard guidelines. The sections were stained with $\mathrm{H} \& \mathrm{E}$ stain and evaluated.

\section{Results}

The overall concordance percentage between frozen section and permanent histological section over a period of one year is $92 \%$ (47/51). Discordance percentage is $2 \%(1 / 51)$ and deferred diagnosis of $6 \%(3 / 51)$. The results were summarised in TABLE 1.

One case from a pancreatic mass lesion was deferred due to inadequate material. Out of the ten cases of thyroid, two from thyroid nodules were reported as of follicular neoplasm (Fig 1 and Fig 2). Permanent sections awaited for a definitive opinion. Reporting o f thyroid lesions in frozen section has inherent limitations because of which the diagnosis was deferred.

The most common indications for frozen section encountered in our institution were for tissue diagnosis and to assess the nodal status in breast and pancreatic malignancies.

The common sites biopsied for frozen section in decreasing order of frequency in our centre is summarised in TABLE 2.

Thirty five out of 51 cases (69\%) were malignant lesions. Of these, 30 cases of breast were infiltrating ductal carcinoma diagnosed by frozen and confirmed later by permanent sections. One case was diagnosed as intraductal papilloma in frozen section. This was diagnosed as mucocele like lesion on permanent section.

In thyroid 8 out of 10 cases were nodular goitre by both frozen and permanent sections and two cases were deferred for want of permanent sections for final diagnosis.

Among five cases of female genital tract two cases were from uterus and two cases from ovary. In uterus one case was endometrial adenocarcinoma (Fig 3 and Fig 4) and one case was leiomyoma by both frozen and permanent sections.

Among 4 cases from lymph node one case was gastric nodal metastasis from gastric adenocarcinoma. Three cases were reactive lymphadenitis. There was $100 \%$ concordance between frozen and permanent sections in these cases.

One case from pancreas was deferred to permanent section diagnosis due to inadequate material. In permanent section it was diagnosed as adenocarcinoma.

Out of two cases from ovary one case was borderline serous papillary neoplasm and another case was endometriosis. There was $100 \%$ concordance between frozen and permanent sections in these cases.

One case was from peritoneal nodule to confirm/exclude malignancy. This was diagnosed as metastatic adenocarcinomatous deposit in both frozen and in permanent tissue sections.

The diagnosis of discordant and deferred cases among frozen and permanent sections are compared and summarised in TABLE 3.

\section{Discussion}

The final results in this present study (concordance 92\%, discordance 2\%, and deferred cases $6 \%$ ) were comparable with results published earlier, where concordance rate was 94 to $96 \%$ and deferred rate was 3-6\%. However, the indication, accuracy and limitations of frozen sections vary with the site of biopsy and have been discussed in detail and published regularly. $[8,9,10,11]$

In this present study, 31 cases $(31 / 51,61 \%)$ of biopsies for frozen section were from breast. It was indicated mainly to establish the diagnosis. In 30 cases, frozen section diagnosis correlated well with permanent diagnosis. One case diagnosed as intraductal papilloma in frozen section was later found to be mucocele like lesion in final permanent sections.

Among 10 cases from thyroid $(10 / 51,20 \%), 8$ cases were nodular goitre and correlated well with permanent section diagnosis. 2 cases were deferred and diagnosed as follicular variant of papillary carcinoma in permanent sections.

These discrepancies were mainly due to inherent limitation of frozen sections in organs like thyroid. Hwang et al and Anton et al mentioned limitations in frozen section evaluation of thyroid. Nuclear features of ground glass appearance as an artefact produced during formalin fixation is a diagnostic feature which is absent in frozen section and alcohol fixed smears. Studies have also confirmed that it is difficult to differentiate benign from malignant follicular lesions at the time of frozen section. It is only a multidisciplinary approach combining frozen with gross examination, cytological and histopathological review which provides the surgeons with the rapid, reliable and cost effective information necessary for optimum patient care. [2,12] 
All cases from female genital tract $(4 / 51,8 \%)$ correlated well with final permanent section diagnosis. One case was borderline serous papillary ovarian neoplasm. According to Coffey et al the concordance rate between frozen and permanent section was lower in mucinous tumours of ovary and mesenchymal tumours of ovary. Extensive sampling from solid portion of ovarian tumour is necessary to rule out invasion in mucinous tumour and to evaluate for the mitotic counts in mesenchymal tumour. [13]

Among 4 lymph nodes, (4/51, 8\%) one was peri gastric nodal metastasis diagnosed correctly and also sub typed as adeno carcinomatous deposit. However, many authors believe that determining the presence of malignancy without sub typing or a judicious deferral can be the best option to decrease the discordance rate.[14]Other 3 cases were reactive lymphadenitis diagnosed correctly.

A retrospective study has documented the important diagnostic errors related to intra operative consultation has divided them into interpretation errors (57\%), sampling error (35\%), and lack of inter departmental communication $(9.5 \%)$. The interpretation errors resulted from artifacts of the freezing procedure, defective sectioning or staining or inexperience on the part of pathologist all of which are avoidable errors. [15, $16,17]$

\section{Tables}

Table 1: Frozen section diagnosis (51) cases compared with diagnosis using permanent sections and tabulated as below

\begin{tabular}{|c|c|c|}
\hline Results & Number & Percentage \\
\hline Concordance & $47 / 51$ & $92 \%$ \\
\hline Discordance & $1 / 51$ & $2 \%$ \\
\hline Deferral & $3 / 51$ & $6 \%$ \\
\hline
\end{tabular}

Table 2: Sites biopsied for frozen section in the order of frequency

\begin{tabular}{|c|c|c|}
\hline Organs & Number of frozen section cases & Number of malignancies in final diagnosis \\
\hline Breast & 31 & 30 \\
\hline Thyroid & 10 & 2 \\
\hline Uterus & 2 & 1 \\
\hline Ovary & 2 & 0 \\
\hline Peritoneum & 1 & 1 \\
\hline Lymph node & 4 & 1 \\
\hline Pancreas & 1 & 0 \\
\hline
\end{tabular}

Table 3: Comparison of discordant and deferred cases among frozen and permanent sections

\begin{tabular}{|c|c|c|}
\hline Organ & Frozen diagnosis & Final diagnosis \\
\hline Breast $(1$ case $)$ & Intraductal papilloma & Mucocele like lesion \\
\hline Thyroid( 2 cases $)$ & Deferred(Indeterminate) & Follicular variant of papillary carcinoma thyroid \\
\hline Pancreas( 1 case $)$ & Deferred( inadequate material) & Adenocarcinoma of pancreas \\
\hline
\end{tabular}

\section{Figures}

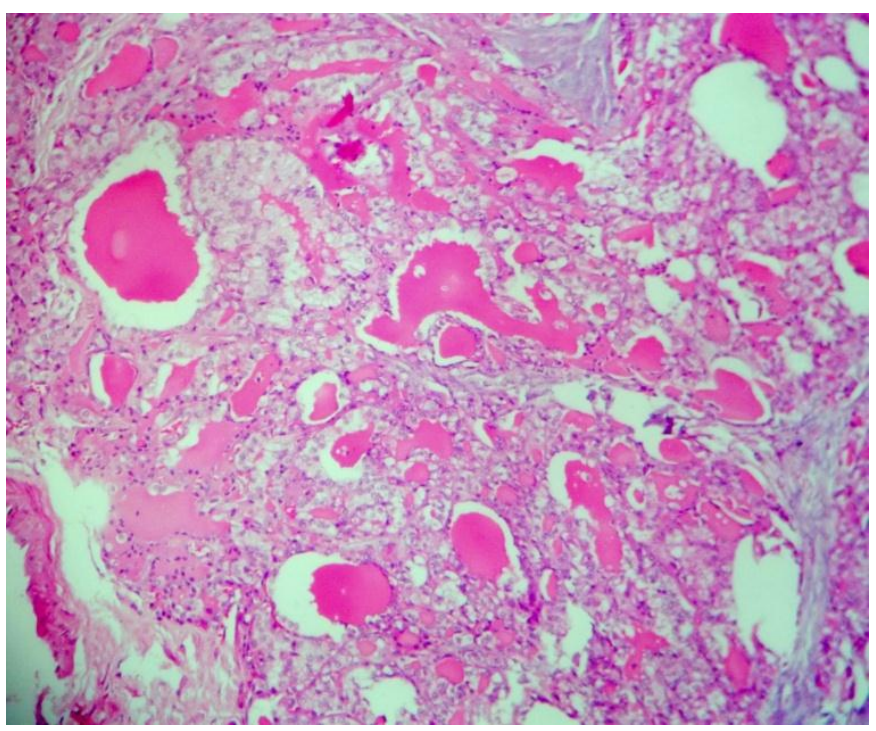

Figure 1: Reported as follicular neoplasm on frozen section. H \& E stain 10X view 


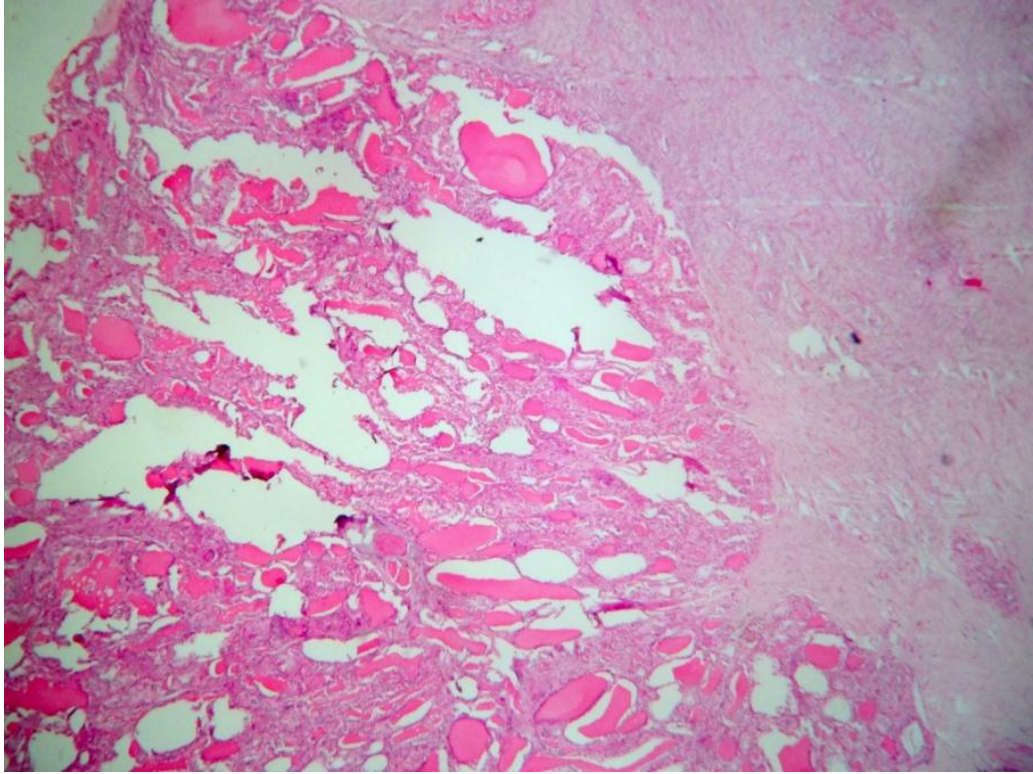

Figure 2: Reported as follicular neoplasm on frozen section. H \& E stain 40X view

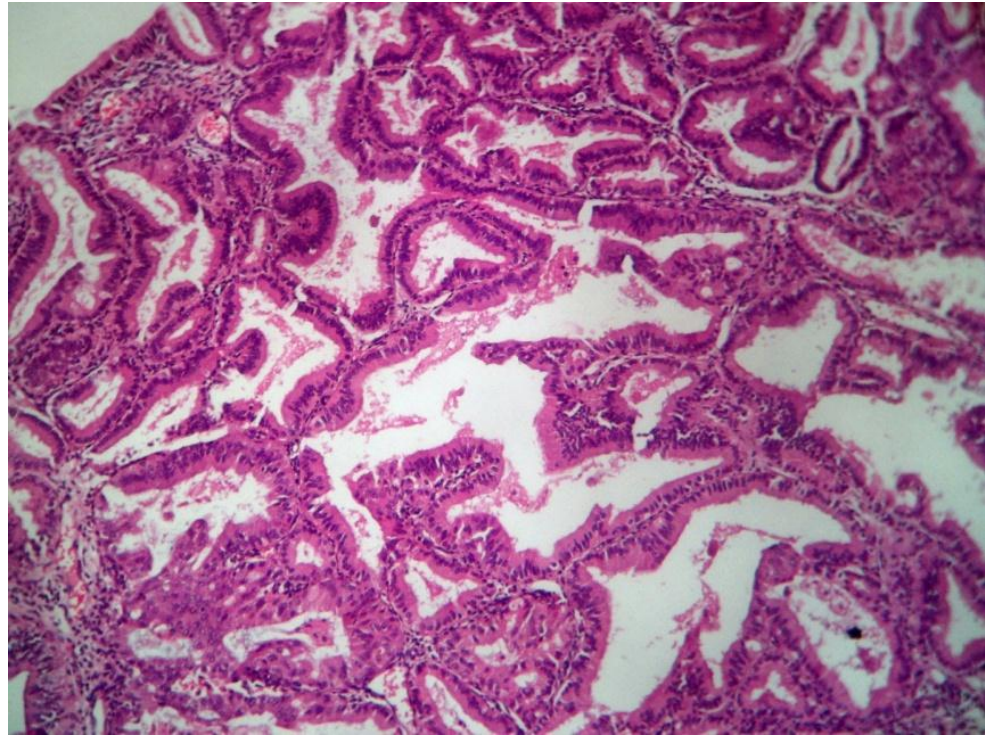

Figure 3: Endometrial adenocarcinoma on frozen section. H \& E stain 10X view

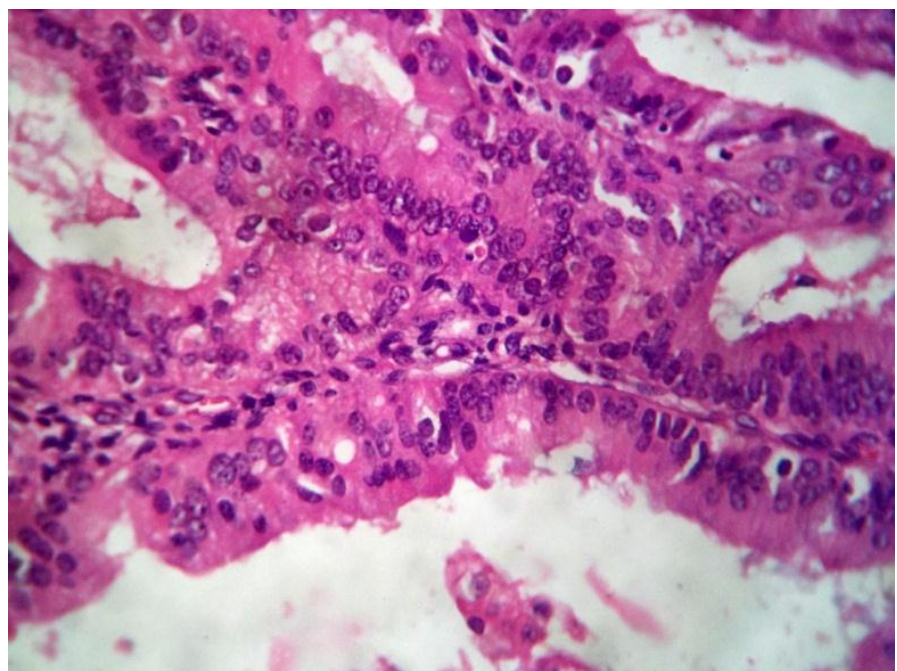

Figure 4: Endometrial adenocarcinoma showing nuclear atypia \& prominent nucleoli on frozen section. H \& E stain $40 \mathrm{X}$ view 


\section{Conclusion}

Frozen section diagnosis is very useful and highly accurate procedure. Methodical macroscopic examination and accurate sampling by pathologist, avoiding technical errors in sectioning and staining, a correct combination of knowledge about the clinical presentation, radiological, biochemical findings and good rapport with the operating surgeon can reduce the limitations and provide rapid, reliable and cost effective details necessary for rapid diagnosis and on table patient management.

\section{References}

[1] Jerome BT. Frozen section and surgical pathologist. Arch Pathol Lab Med 2009; 133:1135-1138.

[2] Hwang TS, Ham EK, Kim CW. et al. An evaluation of frozen section biopsy in 4434 cases. Journal of Korean Medical Science 1987; 2:239-245.

[3] Rebecca D. Folkerth. Smears and Frozen sections in the intra operative diagnosis of central nervous system lesions. Neuropathol 1994:5; 1-18.

[4] Silva EG, Kraemer BB. Intra operative Pathologic Diagnosis. Frozen section and Other techniques.1987

[5] Hull ME, Hunphrey PA, Pfeifer JD. Washington manual of surgical pathology. Elsevier 2006; First Edition: Chapter 51.

[6] Susan CL .Manual of Surgical Pathology. Elsevier 2006; Second Edition: 49-69.

[7] Gephardt GN, Zarbo RJ. International comparison of frozen section consultation. A college of American Pathologists Q-probe study of 90,538 cases in 461 institutions.

[8] Aijaz F,Muzaffar S, Hussainy AS,Pervez S. et al. Intra operative frozen section consultation: an analysis of accuracy in a teaching hospital. J Pak Med Assoc 1993;43(12):253-255.

[9] Novis DA, Gephardt GN, Zarbo RJ. Inter institutional comparison of frozen section consultation in small hospitals: a college of American Pathologists QProbes study of 18,532 frozen section consultation diagnoses in 233 small hospital.Arch Pathol Lab Med 1996;120(12):1087-1093.

[10] Ahmad Z, Barakzai MA, Idrees R.et al. Correlation of intra operative frozen section consultation with the final diagnosis at a referral center in Karachi, Pakisthan. Am J Surg 1993:166(4):424 - 427Dankwa EK, Davies JD. Frozen section diagnosis: an audit. J Clin Pathol 1985; 38:1235-1240

[11] Anton RC, Wheeler TM. Frozen section of Thyroid and Parathyroid Specimens Arch Pathol Lab Med 2005; 129(12): 1575-1578.

[12] Coffey D, Kaplan AL, Ramzy I. Intraoperative Consultation in Gynecologic Pathology. Arch Pathol Lab Med 2005; 129:15441557.

[13] Lechago J. Frozen Section Examination of Liver, gallbladder and Pancreas. Arch Pathol Lab Med 2005; 129(12):1610- 1618.

[14] Nigrisoli E, Gardini G. Quality control of intraoperative diagnosis. Annual review of 1490 frozen sections. Pathologica 1994;86(2):191-195

[15] Rogers C, Klatt EC, Chandrasoma P. Accuracy of frozen section diagnosis in a teaching hospital. Arch Pathol Lab Med 1987; 111:514-517.

[16] Laicirica R. Intraoperative assessment of the Breast: Guidelines and Potential Pitfalls. Arch Pathol Lab Med 2005; 129(12):15651574 . 